\title{
PERFIL EPIDEMIOLÓGICO DA HANSENÍASE NA MICRORREGIÃO DE ARAÇUAÍ E SUA RELAÇÃO COM AÇÕES DE CONTROLE
}

\author{
Epidemiological profile of leprosy at the microregion of araçuaí and its relation with \\ actions control \\ Perfil epidemiológico de la lepra en la micro región de araçuaí y su relación con las \\ acciones de control
}

Francisco Carlos Félix Lana ${ }^{1}$

Ana Paula Mendes Carvalho²

Raquel Ferraz Lopez Davi ${ }^{3}$

\begin{abstract}
RESUMO
A hanseníase representa um problema de saúde pública no Brasil pelos altos índices de prevalência e incidência. 0 objetivo deste estudo é analisar a situação epidemiológica da hanseníase e sua relação com o desenvolvimento das ações de controle na microrregião de Araçuaí. Trata-se de estudo epidemiológico, descritivo, cujos dados foram coletados das fichas de notificação de casos de hanseníase de municípios da microrregião, período 1998-2007, disponibilizados no Sistema de Informação de Agravos de Notificação - SINAN. Foram construídos e analisados indicadores epidemiológicos e operacionais preconizados pelo Ministério da Saúde. Foram notificados 343 casos, resultando em uma detecção geral média de 28,5 casos/100.000 habitantes/ ano. A proporção de casos detectados com grau Il de incapacidade e o predomínio de formas passivas de detecção sugerem diagnóstico tardio e corroboram a importância da integração das ações de controle da hanseníase na atenção básica.
\end{abstract}

Palavras-chave: Hanseníase. Saúde Pública. Atenção Primária à Saúde. Enfermagem

\begin{abstract}
The leprosy represents a public health problem in Brazil, due to the high level of prevalence and incidence. The objective of this study is to analyze the leprosy epidemiological situation and its relation with actions control at the micro-region of Araçuaí. It is an epidemiologic descriptive study and the data were collected from reports of leprosy cases in the cities at the micro-region, during the period of 1998-2007, available in Sistema de Informação de Agravos de Notificação - SINAN. Operational and epidemiological indices were constructed and analyzed based in Ministério da Saúde parameters. 343 cases were notified in the period and the detection rates were 28,5 cases/100.000 habitants/year. The proportion of detected cases with grade II on disability and the prevalence of passive forms of detection suggest a late diagnosis and confirm the importance of integration of leprosy control activities in primary care.
\end{abstract}

Keywords: Leprosy. Public Health. Primary Health Care. Nursing.

\section{Resumen}

La lepra representa un problema de salud pública en Brasil debido a los altos índices de detección y prevalencia. El objetivo de este estudio es analizar la situación epidemiológica de la lepra y su relación con acciones de control en micro región de Araçuaí en el periodo 1998-2007. El estudio es epidemiológico, descriptivo y los dados fueran recogidos de formularios de notificación de casos de lepra de las ciudades de la región, período 1998-2007, disponible en el Sistema de Información de Agravos de Notificación - SINAN. Indicadores operacionales y epidemiológicos fueran construidos y analizados de acuerdo con los parámetros del Ministerio de la Salud. En el periodo, 343 casos de lepra fueran notificados y la detección fue 28,5 casos/100.000 habitantes/año. La proporción de casos detectados con el grado de incapacidad II y la prevalencia de las formas pasivas de detección sugiere un diagnóstico tardío y confirma la importancia de la integración de las actividades de control de la lepra en atención primaria.

Palabras clave: Lepra. Salud Pública. Atención Primaria de Salud. Enfermería.

${ }^{1}$ Enfermeiro. Doutor em Enfermagem. Professor Associado II do Departamento de Enfermagem Materno-Infantil e Saúde Pública da Escola de Enfermagem da Universidade Federal de Minas Gerais - UFMG. Belo Horizonte-MG. Brasil. E-mail: xicolana@ufmg.br, ${ }^{2}$ Enfermeira. Bolsista de Apoio Técnico pela Fundação de Amparo à Pesquisa do Estado de Minas Gerais - FAPEMIG. Escola de Enfermagem da Universidade Federal de Minas Gerais - UFMG. Belo Horizonte- MG. Brasil. E-mail: apmcarv@yahoo.com.br, ${ }^{3}$ Enfermeira. Pesquisadora do Núcleo Interdisciplinar de Estudos e Pesquisa em Epidemiologia - NIEPE - Escola de Enfermagem da Universidade Federal de Minas Gerais- UFMG. Belo Horizonte- MG. Brasil. E-mail: raquelferrazl@gmail.com 


\section{INTRODUÇ̃̃O}

A hanseníase é uma doença infectocontagiosa, de evolução lenta, que se manifesta, principalmente, por sinais e sintomas dermatoneurológicos e apresenta uma tendência crescente nos países em desenvolvimento, sendo considerada um problema de saúde pública no Brasil1.

No ano de 2007 no Brasil, segundo as estatísticas do Ministério da Saúde (MS), o coeficiente de detecção da hanseníase foi de 21,08/100.000 habitantes e o coeficiente de prevalência, de 2,19/10.000 habitantes. Entre os anos de 2001 e 2007 houve uma redução de 19,7\% no coeficiente de detecção da doença, mas, apesar dessa variação, este coeficiente ainda é considerado muito alto segundo parâmetros do MS., ${ }^{1,2}$ 0 coeficiente de prevalência também apresentou grande queda $(47,6 \%)$ entre os anos de 1998 e 2007 sendo 4,18 e 2,19 doentes em cada 10.000 habitantes, respectivamente. ${ }^{1,3}$

A Coordenação Nacional de Controle da Hanseníase assume como objetivo de saúde pública o controle da doença, privilegiando o acompanhamento epidemiológico por meio da detecção de casos novos. ${ }^{1}$ A Organização Mundial de Saúde (OMS), por sua vez, adota dois indicadores brasileiros como prioritários para o controle da doença: a proporção de jovens abaixo de 15 anos entre os novos casos diagnosticados e os registros de pacientes com alto grau de incapacidade. ${ }^{4}$

Em 2007, Minas Gerais registrou 2.235 casos novos de hanseníase, o que resultou em um coeficiente de 11,3 casos/ 100.000 habitantes. Como estratégia para intensificar o controle da hanseníase, a Coordenadoria Estadual de Dermatologia Sanitária da Secretaria de Estado da Saúde de Minas Gerais (SES/MG) tem direcionado suas ações para municípios e regiões considerados prioritários. Dentre essas regiões, encontra-se a microrregião de Araçuaí, com coeficiente de detecção em 2007 de 46,8/100.000 habitantes e seu município sede, Araçuaí, que apresentou uma detecção de $63,74 / 100.000$ habitantes, valores considerados hiperendêmicos. ${ }^{1,2}$

As ações de controle da hanseníase adotadas no Brasil integram atividades de detecção precoce dos casos, tratamento poliquimioterápico, prevenção de incapacidades físicas, vigilância de comunicantes e educação em saúde. As estratégias visam um aumento da cobertura dos serviços de saúde por meio da ampliação da rede de diagnósticos e de atenção ao paciente, mediante a descentralização das atividades para os serviços de atenção básica à saúde. ${ }^{2,5}$

Dentre as ações de controle da doença, tem destaque a reorganização dos serviços de saúde voltada para um rompimento com a tendência da demanda espontânea, de forma a propiciar uma oferta organizada de acordo com as principais necessidades da população atendida. Alguns fatores influenciam a utilização dos serviços de saúde como: a acessibilidade, a existência de especialistas, a competência dos profissionais e 0 estabelecimento de vínculo com o paciente. ${ }^{6,7,8}$
Em um estudo realizado por Amaral e Lana, no ano de 2008, foram identificados diversos fatores que influenciam o controle da hanseníase pelos serviços de saúde, tais como o diagnóstico tardio; 0 abandono dos pacientes ao tratamento; baixos índices de cobertura assistencial, de controle de comunicantes, de esclarecimento sobre a doença e de condições de vida e saúde da população; além do estigma e do preconceito que acometem os portadores da doença. ${ }^{9}$

O Ministério da Saúde preconiza o aumento da cobertura das ações de controle da hanseníase para todas as Unidades de Saúde dos estados e municípios endêmicos, como parte de uma política geral de descentralização, que tem como estratégia o Programa de Saúde da Família (PSF) e o Programa de Agentes Comunitários de Saúde. ${ }^{1}$

Nesse cenário, ressalta-se a importância do trabalho do enfermeiro relacionado ao desenvolvimento das ações de controle por meio da realização de consultas de enfermagem, de assistência domiciliar, de atividades educativas e da busca ativa de casos e da organização e coordenação das ações desenvolvidas. Ferreira e Souza, em um estudo das representações sociais de enfermeiros do PFS sobre a hanseníase, evidenciam a presença do estigma e do medo na fala da maioria dos profissionais entrevistados e chamam atenção para a importância do acompanhamento dos pacientes pela equipe de enfermagem. ${ }^{1,2,10}$

0 presente estudo analisa a situação epidemiológica da hanseníase na microrregião de Araçuaí e sua relação com o desenvolvimento das ações de controle na região, tendo em vista subsidiar a formulação de estratégias para o controle da hanseníase como problema de saúde pública.

\section{MÉTODOS}

Trata-se de um estudo epidemiológico, descritivo, cujo cenário foi a microrregião de Araçuaí, Estado de Minas Gerais, considerando-se a importância que representa para o problema da hanseníase no estado e no Vale do Jequitinhonha, tanto em termos expressos pelos números da doença como de sua posição estratégica na região. Essa microrregião é dividida em seis municípios: Araçuaí, Berilo, Coronel Murta, Francisco Badaró, Jenipapo de Minas e Virgem da Lapa, com uma população total de aproximadamente 89.694 habitantes (estimativa FIBGE/ 2007), e detém alguns dos piores indicadores sociais do estado, o que reflete baixas condições socioeconômicas. ${ }^{11}$

Considerando-se a influência da capacidade operacional dos serviços de saúde e dos programas de controle sobre as informações epidemiológicas relacionadas à hanseníase, 0 período de coleta de dados compreendeu os anos de 1998 a 2007, o que possibilita uma melhor aproximação da realidade da endemia.

Os dados epidemiológicos foram coletados do Sistema de Informação de Agravos de Notificação - SINAN e de Relatórios Técnicos - disponibilizados pela Coordenação 
Estadual de Dermatologia Sanitária. As informações sobre a população residente estratificada por faixas etárias foram obtidas por meio do Censo Demográfico de 2000 e de estimativas populacionais, realizados pela Fundação Instituto Brasileiro de Geografia e Estatística (FIBGE). Conforme recomendação do Ministério da Saúde, optouse por apresentar os coeficientes de detecção por 100.000 habitantes na perspectiva de facilitar a comparação dos coeficientes da hanseníase com outros eventos. ${ }^{1}$

O SINAN representa a fonte nacional de informações sobre agravos de notificação, apresenta ainda algumas limitações relacionadas à fidedignidade dos dados, à duplicidade de registros e à ausência de padronização no lançamento dos dados; entretanto, tem se aperfeiçoado a cada ano e vem se consolidando como uma importante fonte de dados em pesquisas em saúde.

0 tratamento dos dados contemplou a criação de um banco de dados e a construção de indicadores epidemiológicos e operacionais para monitoramento da hanseníase, como forma de investigar a força de morbidade, a magnitude, o perfil epidemiológico e, a partir destas análises, inferir sobre a qualidade das ações de controle dos serviços de saúde. A metodologia de construção dos indicadores foi realizada de acordo com as recomendações do Ministério da Saúde, dispostas na Portaria Conjunta n⿳0 125 de 26 de março de 2009. ${ }^{2}$

Os indicadores epidemiológicos e operacionais utilizados foram: coeficiente de detecção anual de casos novos de hanseníase por 100.000 habitantes; coeficiente de detecção anual de casos novos de hanseníase na população de 0 a 14 anos por 100.000 habitantes; proporção de casos novos de hanseníase com o grau de incapacidade física avaliado no diagnóstico; proporção de casos de hanseníase com grau 2 de incapacidade física no momento do diagnóstico, entre os casos novos detectados e avaliados no ano; proporção de casos curados no ano com grau de incapacidade física avaliado; proporção de casos de hanseníase em abandono de tratamento entre os casos novos diagnosticados nos anos das coortes. ${ }^{2}$ Outros parâmetros utilizados para a análise dos dados foram o modo de detecção, a forma clínica, a classificação operacional dos casos novos de hanseníase e a unidade de saúde de diagnóstico dos casos notificados.

A análise dos dados foi realizada por meio da avaliação dos indicadores de acordo com os parâmetros e políticas para o controle da hanseníase do Ministério da Saúde, comparando a situação epidemiológica dos municípios estudados com outros municípios da mesma região, com o estado e país nos diversos anos estudados. ${ }^{2}$

A referida pesquisa atende a resolução n. ${ }^{0} 196$ de 10 de outubro de 1996, do Conselho Nacional de Saúde, que dispõe sobre aspectos éticos de pesquisas que envolvem seres humanos e foi aprovada pelo Comitê de Ética em Pesquisa da Universidade Federal de Minas Gerais, segundo parecer n ${ }^{\circ}$. ETIC 149/07 em 27 de julho de 2007. Para a construção do banco de dados foram omitidas informações relacionadas à identificação pessoal dos casos notificados, como forma de preservar o sigilo e 0 anonimato dos indivíduos.

\section{RESULTADO E DISCUSSÃO}

No período de 1998 a 2007 foram notificados 343 casos de hanseníase na microrregião de Araçuaí, o que resulta em uma detecção média no período de 28,5 casos por 100.000 habitantes. Segundo os parâmetros do Ministério da Saúde ${ }^{2}$, esse coeficiente é considerado muito alto, o que ratifica sua condição de área prioritária para o controle da endemia em Minas Geras. ${ }^{5}$

A Tabela 1 apresenta o número de casos de hanseníase registrados na microrregião, segundo município e ano de notificação, e o coeficiente médio de detecção. Nota-se que há um acúmulo de casos no município de Araçuaí, que responde por 71,7\% $(n=243)$ do total da microrregião, embora represente aproximadamente $40 \%$ da população total. Paradoxalmente, os municípios de Berilo e Virgem da Lapa, que juntos possuem aproximadamente $30 \%$ da população, foram responsáveis por apenas $5,9 \%$ das notificações $(n=20)$. Esses dados sugerem que os serviços de saúde do município de Araçuaí estariam mais estruturados, com maior capacidade operacional e, portanto, foram capazes de identificar um maior número de casos de hanseníase presente na população. $^{9}$

Cabe ressaltar que em municípios com pequenas populações, poucos casos novos resultam em altos coeficientes, o que pode mascarar a real situação epidemiológica. ${ }^{12}$ Tal fato foi observado no município de Coronel Murta, que no ano de 2000 detectou 1 caso, coeficiente de 9,92/100.000 hab., e no ano seguinte apresentou 17 casos novos, o que resultou em um coeficiente de 186,49/100.000hab. 0 município de Jenipapo de Minas apresentou coeficientes nulos em quatro anos do estudo, e em 2002, a detecção de 5 casos representou um coeficiente de 78,2/100.000 habitantes.

A permanência de municípios com coeficientes de detecção de hanseníase muito baixos ou nulos, localizados em regiões consideradas hiperendêmicas, pode estar associada à baixa integração das ações de controle da doença nos serviços da atenção básica, à falta de engajamento dos gestores e de capacitação para os profissionais de saúde. ${ }^{8,9}$ 
Tabela 1. Número de casos e coeficiente médio de deteç̧ão de hanseníase por 100.000 habitantes na microrregião Araçuaí, segundo município de residência. Período 1998-2007

\begin{tabular}{ccccccccccccc}
\hline MUNICíPIO & 1998 & 1999 & 2000 & 2001 & 2002 & 2003 & 2004 & 2005 & 2006 & 2007 & TOTAL & $\begin{array}{c}\text { Coef.Médio } \\
\text { de Deteçãa }\end{array}$ \\
\hline Araçuaí & 25 & 23 & 23 & 19 & 23 & 29 & 32 & 21 & 28 & 23 & 246 & 67,9 \\
$\quad$ Berilo & 0 & 0 & 1 & 0 & 0 & 1 & 2 & 1 & 0 & 1 & 6 & 4,64 \\
Coronel Murta & 0 & 1 & 1 & 17 & 5 & 4 & 6 & 1 & 2 & 7 & 44 & 48,08 \\
$\quad \begin{array}{c}\text { Francisco } \\
\text { Ba daró }\end{array}$ & 0 & 0 & 0 & 2 & 2 & 2 & 1 & 1 & 3 & 9 & 20 & 19,4 \\
$\begin{array}{c}\text { Jenipapo de } \\
\text { Minas }\end{array}$ & 0 & 0 & 0 & 1 & 5 & 4 & 1 & 1 & 1 & 0 & 13 & 20,52 \\
Virgem da Lapa & 1 & 2 & 0 & 0 & 0 & 4 & 2 & 1 & 2 & 2 & 14 & 10,32 \\
\hline Total & 26 & 26 & 25 & 39 & 35 & 44 & 44 & 26 & 36 & 41 & 343 & \\
\hline
\end{tabular}

Fonte: Fichas de Notificação de Casos de Hanseníase - SINAN

0 Gráfico 1 apresenta os coeficientes de detecção geral e em menores de 15 anos da hanseníase na microrregião, segundo ano de notificação. Observa-se um aumento não linear na detecção da doença, considerando os coeficientes de detecção do início e do final do período, 29,1/100.000 habitantes e 46,8/100.000 habitantes, respectivamente.

0 coeficiente de detecção de hanseníase em menores de 15 anos, principal indicador epidemiológico para o controle da doença, é utilizado como forma de avaliar a força de transmissão recente da endemia e sua tendência, uma vez que o bacilo causador da doença possui um longo período de incubação. ${ }^{1}$ Este coeficiente também apresentou oscilações não lineares no período estudado, variando de 1,83/100.000 habitantes, em 1998 a 4,22/100.000 habitantes, em 2007. Cabe ressaltar que, apesar das oscilações encontradas, os coeficientes de detecção estão sempre em patamares muito elevados.

Gráfico 1. Distribuição do coeficiente de detecção geral de hanseníase e na população menor de 15 anos por 100.000 habitantes, na microrregião Araçuaí, segundo ano de notificação

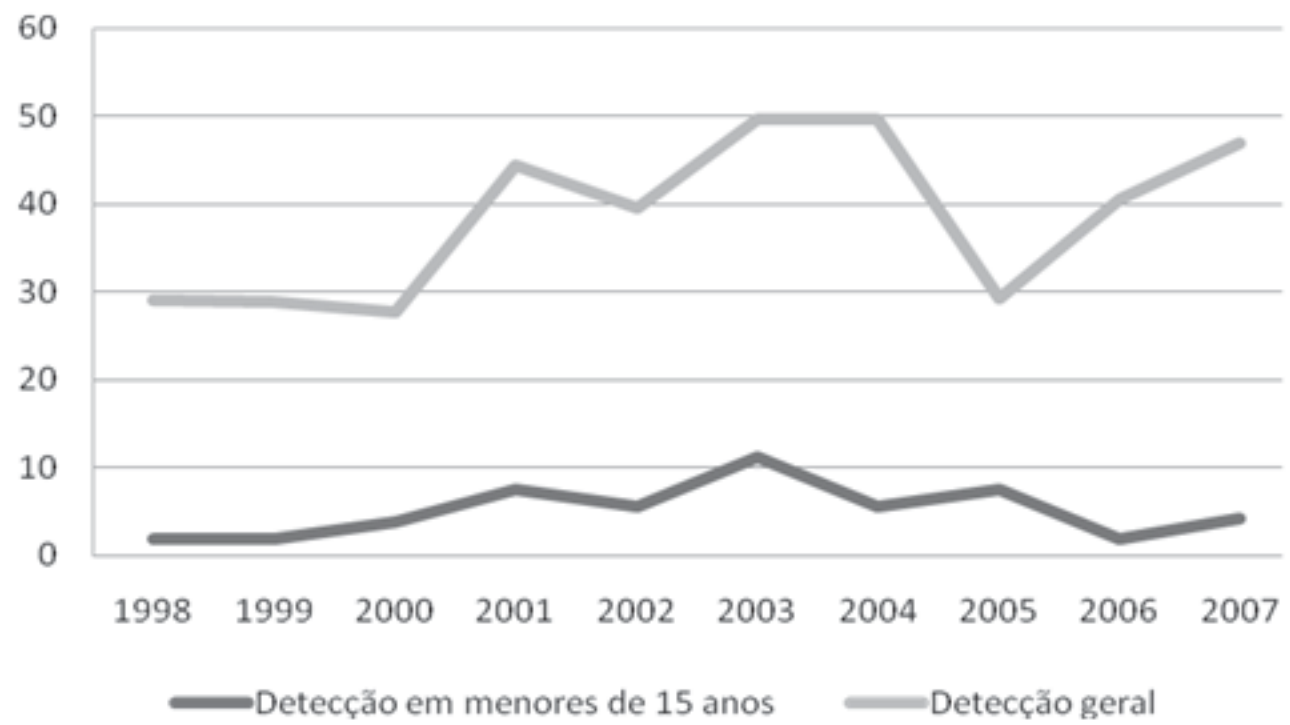

Fonte: Fichas de Notificação de Casos de Hanseníase - SINAN

As formas clínicas multibacilares (dimorfa e virchowiana), consideradas as principais fontes de infecção da hanseníase, predominaram com $63,2 \%$ do total de casos. Dos 217 pacientes multibacilares, $27,2 \%$ apresentavam grau I de incapacidade física e $11,9 \%$ apresentavam grau II no momento do diagnóstico. Segundo alguns autores, essa situação indica que o diagnóstico tem ocorrido tardiamente, colaborando para a manutenção da cadeia de transmissão. 9, ${ }^{92,13}$ Para Oliveira, ${ }^{3}$ no entanto, este fato seria um sinal de estabilização da endemia ou de ocorrência de baixa prevalência, uma vez que apenas os indivíduos mais susceptíveis estariam adoecendo.
As incapacidades físicas e deformidades estão entre os principais agravos provocados pela hanseníase, uma vez que podem ocasionar ou agravar os processos de exclusão social e interferir nas atividades laborais dos pacientes, o que afeta negativamente a qualidade de vida dos indivíduos. ${ }^{14,15}$

Na microrregião, foram diagnosticados 255 pacientes $(74 \%$ do total de casos) com grau zero - sem acometimentos físicos - 61 pacientes (17,8\% do total de casos) com grau I de incapacidade física - diminuição ou perda da sensibilidade nos olhos, nas mãos e nos pés - e 27 pacientes (7,9\% do total de casos) com grau II - alterações visuais e lesões tróficas e/ou traumáticas nas mãos enos pés.? 
Deve-se ressaltar que a proporção de casos diagnosticados com grau Il de incapacidade física é utilizada como forma de avaliar a efetividade de detecção precoce de casos de hanseníase. 0 índice de 7,9\% de detecção de casos com esse grau de incapacidade física na microrregião está acima dos parâmetros preconizados, o que indica a ocorrência de diagnóstico tardio. ${ }^{2}$ A realização de diagnóstico tardio sugere que os serviços de saúde da região não estariam sendo capazes de captar todos os doentes existentes, o que contribuiria para a permanência de casos não diagnosticados (prevalência oculta) e a continuidade da cadeia de transmissão. ${ }^{2}$

Um estudo realizado nas cinco microrregiões do Vale do Jequitinhonha (Almenara, Araçuaí, Capelinha, Diamantina e Pedra Azul), em 2008, aponta para o elevado percentual de casos de hanseníase diagnosticados com grau ll de incapacidade física na região. Ressalta-se a passividade dos serviços de saúde na implementação de estratégias de prevenção e controle da doença e a necessidade de intensificação dessas ações. ${ }^{13}$

A avaliação dos casos quanto às incapacidades físicas possibilita uma estimativa da situação epidemiológica da doença de forma a subsidiar a implementação de ações de controle. Todos os casos registrados na microrregião tiveram o grau de incapacidade física avaliado no diagnóstico, e apenas 44,3\% dos pacientes foram avaliados no momento da alta por cura. Dos 152 casos que foram avaliados no diagnóstico e na alta, $13,8 \%(n=21)$ tiveram redução no grau de incapacidade física, $80,9 \%(n=123)$ mantiveram o mesmo grau e $5,3 \%(n=8)$ aumentaram a incapacidade física após a conclusão do tratamento.

Essa situação aponta para uma priorização da avaliação de incapacidades no momento do diagnóstico e um acompanhamento deficiente dos serviços de saúde com relação ao desenvolvimento de incapacidades físicas durante 0 tratamento. Deve-se destacar que a precariedade desses dados dificulta a realização de uma análise verossímil sobre a evolução do grau de incapacidade física nessa microrregião.

0 modo de descoberta de um caso novo também pode ser associado à forma como os serviços de saúde se organizam para prestar assistência de saúde em hanseníase. Neste estudo, $87,5 \%$ dos casos foram descobertos por meio de formas passivas de detecção - demanda espontânea ou encaminhamento. Esse quadro evidencia a baixa realização de busca ativa de casos novos nos serviços de saúde de atenção básica na microrregião Araçuaí.

Segundo estudo realizado por Lanza ${ }^{8}$ na microrregião de Almenara, que apresenta características semelhantes à microrregião de Araçuaí, a organização dos serviços de saúde está estruturada no modelo clínico, que prioriza o atendimento individual do doente, sendo que as práticas de saúde relacionadas à abordagem coletiva como a busca ativa e a vigilância epidemiológica ainda são muito pontuais.

Para Amaral ${ }^{9}$, a passividade dos métodos de detecção pode estar relacionada à diminuição do coeficiente de deteç̧ão e contribui para aumentar o número de casos não diagnosticados, mascarando a real situação epidemiológica da hanseníase. Em contrapartida, Pereira et a $/{ }^{16}$ veem aspectos positivos no comparecimento voluntário dos doentes às unidades de saúde, uma vez que isso refletiria uma população bem informada em relação aos sinais e sintomas da doença.

Em Araçuaí, $69,9 \%$ dos casos $(n=172)$ foram notificados na Policlínica Municipal entre os anos de 1998 e 2007, sendo que a partir de 2001 todos os casos foram notificados nessa unidade, o que sugere uma centralização do diagnóstico de hanseníase em uma unidade de atenção secundária.

Na microrregião de Almenara, observou-se que as unidades de Programa de Saúde da Família ainda não se encontram estruturadas para realizar as ações de controle da doença ${ }^{8}$. Vários fatores estão relacionados a esta desestruturação, como a alta rotatividade dos profissionais, a não sensibilização e comprometimento de gestores e profissionais de saúde com o problema da hanseníase e deficiências na capacitação. ${ }^{8}$

Outros estudos realizados em municípios dos Estados de São Paulo e do Rio de Janeiro ratificam o fato de que os serviços de saúde de menor complexidade não estão organizados para realizar ações de controle de hanseníase e têm encaminhado os casos suspeitos para as unidades de referência. ${ }^{15,16}$

A descentralização das ações de controle da hanseníase é essencial para melhorar o acesso da população ao serviço. A atenção básica deve incorporar em seu elenco de atividades a disponibilização de recursos e a divulgação de informações relacionadas aos sinais e sintomas, ao diagnóstico, ao tratamento poliquimioterápico, à avaliação e à prevenção de incapacidades, à busca ativa e ao controle de comunicantes. Os centros de referência, como as policlínicas, devem ser reorganizados para prestarem assistência às complicações que foram referenciados pela atenção básica e promoverem a educação continuada das equipes de saúde. ${ }^{8,15}$

\section{CONCLUSÃO}

No que pese as limitações da utilização de dados secundários, foi possível realizar a análise epidemiológica da hanseníase na microrregião de Araçuaí, bem como inferir sobre a qualidade das ações de controle empreendidas pelos serviços de saúde dos municípios. Observa-se que a estrutura e a organização dos serviços de saúde têm influência importante no desenho da atual situação epidemiológica da hanseníase nessa microrregião.

0 alto coeficiente de detecção da hanseníase encontrado na microrregião ratifica a condição de área prioritária para o controle da doença. Além disso, o aumento do número de casos em indivíduos menores de quinze anos e o predomínio das 
formas multibacilares, responsáveis pela manutenção da cadeia de transmissão, sugerem expansão da endemia.

Os altos índices de casos que apresentavam incapacidades físicas no momento da deteç̧ão apontam para a realização do diagnóstico tardio que, associado à descoberta de casos novos predominantemente por formas passivas e a centralização do diagnóstico na atenção secundária, contribuem para a manutenção da prevalência oculta.

Diante deste cenário, evidencia-se a necessidade de intensificar o desenvolvimento das ações de controle da hanseníase na microrregião, facilitando o acesso ao diagnóstico e ao tratamento. Para tanto, é importante a reorganização do processo de trabalho de forma a integrar as ações de controle aos serviços de atenção básica, sobretudo nas Equipes de Saúde da Família, com ênfase na abordagem coletiva.

Os resultados evidenciam a necessidade de realização de novos estudos que visem compreender melhor a influência da organização dos serviços de saúde e da dinâmica dos processos de trabalho de forma a subsidiar o desenvolvimento de outras estratégias para o controle da hanseníase.

\section{REFERÊNCIAS}

1. Ministério da Saúde (BR). Secretaria de Vigilância em Saúde. Vigilância em Saúde: situação epidemiológica da hanseníase no Brasil. Brasília(DF); 2008.

2.Secretaria deVigilância em Saude. Portaria Conjunta n. 125, de 26 de março de 2009. Define as ações de controle da hanseníase e dá outras providencias. Diário Oficial [da] Republica Federativa do Brasil, Poder Executivo,Brasilia(DF) 2007 mar 27; Secão 1: 73.

3 - Oliveira MLW, Motta CP. A hanseníase como problema de saúde pública. In: Lombardi C, organizador. Hanseníase: epidemiologia e controle. São Paulo(SP): Imprensa Oficial do Estado; 1990. p. 13-32.

4.Ministério da Saúde (BR). Portal da saúde. Notícias: OMS adota indicadores do Brasil para controlar hanseníase. [citado 2009 ago 06]. Disponível em: http://portal.saude.gov.br/portal/aplicacoes/noticias/ default.cfm?pg=dspDetalheNoticia\&id_area=124\&CO_NOTICIA=10441

5.Secretaria de Estado da Saúde (MG). Coordenadoria Estadual de Dermatologia Sanitária. Seminário Estadual 2008: Mobilizando parceiros para o controle da hanseníase. Belo Horizonte (MG); 2008.

6. Lapa TM, Albuquerque MFPM, Carvalho MS, Silveira JJC. Análise da demanda de casos de hanseníase aos serviços de saúde através do uso de técnicas de análise espacial. Cad Saude Publica. 2006 dez; 22(12): 2575-583.

7. Adami NP. Acesso, utilização e aceitação dos serviços de dermatologia de um Centro de Saúde Escola sob o modo de ver dos hansenianos. Rev Latino-am Enfermagem. 1993 jul; 1(2): 53-67.

8. Lanza FM. Tecnologia do processo de trabalho em hanseníase: Análise das ações de controle na microrregião de Almenara, Minas Gerais [dissertação]. Belo Horizonte (MG): Escola de Enfermagem, Universidade Federal de Minas Gerais; 2009.
9.Amaral EP, Lana FCF. Análise espacial da hanseníase na microrregião de Almenara, MG, Brasil. Rev Bras Enferm. 2008 nov; 61 ( n esp): 701-07.

10.Ferreira AMR, Souza MJ. Representações sociais de enfermeiros do programa saúde da família sobre a hanseníase: uma contribuição para a enfermagem. Esc Anna Nery. 2004 dez; 8(3): 448-54.

11. Instituto Brasileiro de Geografia e Estatística - IBGE. Contagem da população 2007. Rio de Janeiro (RJ); 2007.

12.Lastória JC, Putinatti SMA. Utilização de busca ativa de hanseníase: relato de uma experiência de abordagem na detecção de casos novos. Hansen Int. 2004 jan/jun; 29(1): 6-11.

13.Lana FCF, Amaral EV, Lanza FM, Saldanha ANSL. Physical disabilities resulting from hansen's disease in Vale do Jequitinhonha, Minas Gerais. Rev Latino-am Enfermagem. 2008 nov/dez; 15(6): 993-97.

14.Duarte MTC, Ayres JA, Simonetti JP. Socioeconomic and demographic profile of leprosy carriers attended in nursing consultations. Rev Latinoam Enfermagem. 2007 set/out; 15(esp): 774-79.

15.Moreira TMA. Avaliação da descentralização das ações programáticas de hanseníase: um estudo de caso [tese]. Rio de Janeiro (RJ): Escola Nacional de Saúde Pública, Fundação Oswaldo Cruz; 2002.

16.Pinto Neto JM, Villa TCS. Características epidemiológicas dos comunicantes de hanseníase que desenvolveram a doença, notificados no Centro de Saúde de Fernandópolis (1993 a 1997). Hansen Int.1999 jul/dez; 24(2): 129-36.

17.Pereira AJ, Helene LMF, Pedrazini ES, Martins CL, Vieira CSCA. Atenção básica de saúde e a assistência em hanseníase em serviços de saúde de um município do Estado de São Paulo. Rev Bras Enferm. 2008 nov; 61(n esp): 718-26. 